\title{
Range Extension of Passive Wake-up Radio Systems through Energy Harvesting
}

\author{
Li Chen, ${ }^{*}$ Stephen Cool, ${ }^{*} \mathrm{He} \mathrm{Ba},{ }^{*}$ Wendi Heinzelman, ${ }^{*}$ Ilker Demirkol, ${ }^{\dagger \S}$ \\ Ufuk Muncuk, ${ }^{\ddagger}$ Kaushik Chowdhury, ${ }^{\ddagger}$ and Stefano Basagni ${ }^{\ddagger}$ \\ ${ }^{*}$ Department of Electrical and Computer Engineering, University of Rochester, Rochester, NY, USA \\ Email: \{lichen@ece, scool@u,ba@ece,wheinzel@ece\}.rochester.edu \\ ${ }^{\dagger}$ Department of Telematics Engineering, Universitat Politecnica de Catalunya, Barcelona, Spain \\ §Fundacio i2CAT, Barcelona, Spain. Email: ilker.demirkol@entel.upc.edu \\ $\ddagger$ Department of Electrical and Computer Engineering, Northeastern University, Boston, MA, USA \\ Email: \{umuncuk@coe,krc@ece, basagni@ece\}.neu.edu
}

\begin{abstract}
Use of a passive wake-up radio can drastically increase the network lifetime in a sensor network by reducing or even completely eliminating unnecessary idle listening. A sensor node with a wake-up radio receiver (WuRx) can operate in an extremely low power sleep mode until it receives a trigger signal sent by a wake-up radio transmitter (WuTx). After receiving the trigger signal, the attached WuRx wakes up the sensor node to start the data communication. In this paper, we implement and compare the performance of three passive wake-up radio-based sensor nodes: 1) WISP-Mote, which is a sensor mote that employs an Intel WISP passive RFID tag as the WuRx; 2) EH-WISP-Mote, which combines a novel energy harvester with the WISP-Mote; and 3) REACH-Mote, which uses the energy harvester circuit combined with an ultra-low-power pulse generator to trigger the wake-up of the mote. Experimental results show that the wake-up range and wake-up delay for the EH-WISP-Mote are improved compared with the WISP-Mote, while providing the ability to perform both broadcast-based and ID-based wake-ups. On the other hand, the REACH-Mote, which can only provide broadcast-based wake-up, can achieve a much longer wake-up range than any known passive wake-up radio to date, achieving feasible wake-up at a range of up to $37 f t$.
\end{abstract}

\section{INTRODUCTION}

A wireless sensor network (WSN) consists of a set of sensor nodes organized into a cooperative network. Each sensor node may contain processing units (e.g., a microprocessor, some DSP processors, or even a CPU), multiple types of memory to store programs, collected data, or even an operating system, multiple sensors to obtain data from the environment (e.g., a temperature sensor, a humidity sensor, etc.), a power source (batteries or an energy harvesting component) and a wireless transceiver. There are typically one or more data sinks in the network that must gather the data sensed by the nodes.

Prolonging the network lifetime is one of the key challenges in WSNs. Most sensor nodes are battery powered, and due to the size and/or cost constraints, the battery life of each sensor node is limited. As a WSN is a self-organizing ad-hoc network, the network lifetime is affected by the communication traffic

\footnotetext{
${ }^{1}$ This research was funded in part by the National Science Foundation under research grant CNS-1143662 and in part by the Spanish Government, MICINN, under research grants TEC2009-11453 and FEDER.
}

of the network, the communication protocols, and the battery life of each sensor node.

One method to improve the network lifetime is to duty cycle the sensor nodes, such that the nodes are put into a sleep state periodically. However, this approach requires accurate synchronization among the sensor nodes, as one node can only transmit data to another while both sensor nodes are active. In addition, unnecessary idle listening by receivers that are not the target of a transmission wastes energy. Low power listening (LPL), also called preamble sampling, is another solution to improve network lifetime for WSNs. LPL aims to reduce idle listening in asynchronous protocols by shifting the burden of synchronization to the sender. However, idle listening is not eliminated. For both synchronous duty cycling and low power listening approaches, reducing the duty cycle of a node can increase its lifetime at the cost of increasing the delay in data delivery and reducing the packet delivery ratio.

The use of a wake-up radio (WuR) is another approach to increase network lifetime by employing additional wakeup radio hardware. A wake-up transmitter (WuTx) initializes the transmission by sending a wake-up signal. When a wakeup receiver $(\mathrm{WuRx})$ receives this signal, it will trigger the sensor node to awaken it from its sleeping mode to start data communication. This on-demand wake-up can save the energy wasted by idle listening. Moreover, the wake-up radio approach has potential advantages over duty cycling in terms of delay, collision, overhead and protocol complexity.

There are two types of wake-up radios being developed: active wake-up radios and passive wake-up radios. An active wake-up radio sensor node requires a power supply for the wake-up circuit, but usually has a relatively long wake-up range. On the other hand, a passive wake-up radio sensor node only utilizes the energy harvested from the wake-up radio and does not dissipate any energy from the battery. However, as the energy harvested by the wake-up circuit is limited, passive wake-up radio sensor nodes operate over a shorter range of distances compared to active wake-up radio sensor nodes.

RFID technology is one of the feasible approaches to achieve a passive wake-up radio, as RFID has a well-defined communication protocol and uses energy harvesting to enable 
this communication. However, an RFID receiver (i.e., a tag) used in a wake-up radio sensor node can only achieve a relatively short wake-up range compared to the communication range of the sensor node. As a sensor node with a WuRx can start communication only after it is awoken, a longer wakeup range can improve the communication efficacy of a WSN equipped with WuRs. In addition, a longer range enables a wider range of WSN applications to benefit from the use of WuRs. It is, therefore, necessary to explore further passive wake-up radio sensor nodes and to develop an extended range wake-up radio sensor network; this is the goal of the work described in this paper.

In our previous work, we developed one of the first implementations of a passive wake-up radio sensor node. This node, called a WISP-Mote [1], combined an Intel passive RFID device (a WISP) with a Tmote Sky mote. We determined the performance of the WISP-Mote through field experiments, and we described the advantages of using WISP-Motes compared with duty cycling through simulations based on the field test measurements. The WISP-Mote is able to provide both broadcast-based wake-up (waking up all nodes in range of the WuTx) and ID-based wake-up (waking up a particular node by sending the ID of that node in the wake-up signal).

Clearly, one way to extend the range of a passive wake-up radio sensor node is to provide improved energy harvesting efficiency. Following this reasoning, we combined a novel energy harvesting circuit we developed [2] with our WISPMote to create a new device that we call an EH-WISP-Mote (Enhanced-WISP-Mote). In this paper, we provide field experiments that show the EH-WISP-Mote can, indeed, improve the wake-up range and reduce the wake-up delay compared with the WISP-Mote. However, the EH-WISP-Mote contains extra hardware that is necessary for ID-based wake-up, to decode a particular ID in the wake-up signal, that is not required for broadcast-based wake-up.

Hence, in this paper we also describe the design of a novel wake-up radio sensor node, which we call REACHMote (Range EnhAnCing energy Harvester-Mote), composed of the energy harvesting circuit [2] and an ultra low power wake-up pulse generator that uses a Maxim Integrated chip [3] as the WuRx, and a Tmote Sky mote as the wireless sensor node. We implemented the REACH-Mote and characterized its performance in terms of wake-up delay over a range of distances. The field test results show that the REACH-Mote can achieve a feasible wake-up range of about $37 \mathrm{ft}$, which is almost double that of any known passive wake-up radio.

The remainder of this paper is orgnized as follows. In Section II, we present a survey of related work. The description of our novel high efficiency energy harvesting circuit and the design of the EH-WISP-Mote are provided in Section III. Section IV describes the hardware design of our new passive wake-up radio sensor node, the REACH-Mote. Section V presents results from field experiments using the three passive wake-up radio designs (WISP-Mote, EH-WISP-Mote and REACH-Mote), and conclusions are drawn in Section VI.

\section{RELATED WORK}

Most research on wake-up radio sensor nodes focuses on active wake-up radios, as these can provide a relatively long wake-up range [4], while oftentimes providing lower energy consumption than duty cycling. Some innovative ideas are proposed for active wake-up radio designs to reduce the energy consumption as low as possible. Sanchezet et al. proposed an RFID-based wake-up radio for wireless sensor networks [5]. The circuit they proposed can reach a wake-up range of $49.2 \mathrm{ft}$. However, the circuit is an active circuit with $8.7 \mu \mathrm{W}$ energy consumption in waiting mode [6], and $20.7 \mu \mathrm{W}$ during the wake-up signal processing mode. Kolinko et al. proposed and implemented a semi passive wake-up radio that consumes $20 \mu \mathrm{W}$ and can achieve a range of $700 \mathrm{ft}$ with a $+30 \mathrm{dBm}$ wake-up transmitter. As low as the energy consumption of these wake-up radios are, they still require battery energy to operate.

For passive wake-up radio sensor nodes, which is the target area of this paper, there are few existing approaches in the literature. To the best of our knowledge, the only hardware implementation of a passive wake-up radio is our WISPMote [1]. A WISP-Mote is composed of a WISP, which is a programable RFID tag with a digital I/O to output the wake-up trigger, and a Tmote Sky sensor node. The WISP-Mote can be awakened by an Impinj RFID reader [7] at a maximum distance of approximately $13 \mathrm{ft}$. We determined the energy consumption and also evaluated via simulation the network performance when using WISP-Motes in a network with either a mobile sink or mobile sensors. In this paper, we extend the design of the WISP-Mote to include an energy harvesting circuit, and we also design a completely new passive wakeup radio sensor node that uses the energy harvesting circuit combined with some additional circuitry to create the wake-up trigger for the mote.

Better energy harvesting efficiency will lead to better performance for passive wake-up radio sensor nodes. Energy harvesting, a well investigated area in many fields of science and engineering, ranging from civil and wind engineering to automotive applications, has been recently investigated also more specifically for WSNs. In fact, the development of small factor harvesters for many different energy sources combined with new and efficient wake-up techniques as described here, makes passive wake-up radio WSNs the technology of choice for applications requiring the network to function for years or even decades. Energy harvesting techniques for different sources, devices, and new protocol designs for WSNs with energy harvesting nodes are surveyed by Basagni et al. in [8].

\section{Energy Harvesting Circuit Design}

Our RF energy harvesting circuit enhances the wake-up ability of the WISP, by increasing its wake-up distance. In this section, we describe the general design of the energy harvesting circuit and interfacing principles, as well as motivate the choice of specific circuit component. 


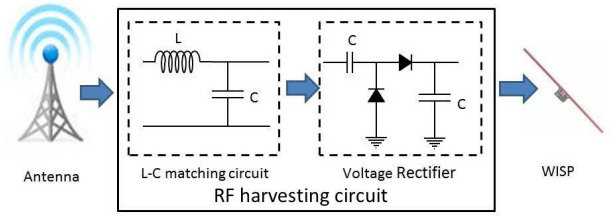

Fig. 1. Architectural view of the circuit and connections.

\section{A. Selection of Circuit Components}

The overall aim of our design is to maximize the energy conversion from the front-end antenna to the WISP-Mote. For this aim, as shown in Fig. 1, we carefully tune a matching circuit to balance the input impedance seen from the antenna side with the circuit load (i.e., the WISP and Tmote combination), as well as use a voltage rectifier that also functions as a multiplier. The multiplier is based on the classical Dickson's voltage multiplier circuit (Fig. 2), which has a number of stages connected in parallel, each stage being a series combination of a diode and a capacitor. The advantage here is that because the capacitors appear in parallel with respect to each other, the effective circuit impedance is reduced. Hence, this makes the task of matching the antenna side to the load side simpler.

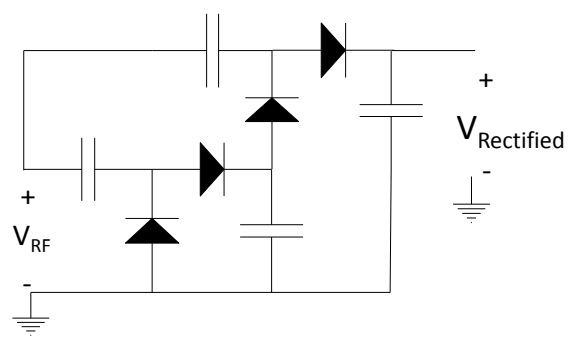

Fig. 2. Dickson diode based multiplier.

As the peak voltage of the AC signal obtained at the antenna is generally much smaller than the diode threshold [9], diodes with lowest possible turn on voltage are preferable. Moreover, since the energy harvesting circuit operates in the high $\mathrm{MHz}$ range, diodes with a very fast switching time need to be used. Schottky diodes use a metal-semiconductor junction instead of a semiconductor-semiconductor junction. This allows the junction to operate much faster, and gives a forward voltage drop of as low as $0.15 \mathrm{~V}$. We employ diodes from Avago Technologies, HSMS-2852 that have a turn-on voltage of $150 \mathrm{mV}$, measured at $0.1 \mathrm{~mA}$, because this specific diode is suitable for operating in the low power region, typically considered as the range of power between $-20 \mathrm{dBm}$ and $0 \mathrm{dBm}$.

The selection of the number of multiplier stages has a major influence on the output voltage of the energy harvesting circuit. While the output voltage is directly proportional to the number of stages used in the energy harvesting circuit, it also reduces progressively the current drawn by the load, which in turn impacts the overall charging time. We set the number of stages to 10 as this ensures sufficient output voltage of the circuit to drive the WISP at $915 \mathrm{MHz}$.

\section{B. Optimization Framework and Fabrication}

The selection of the precise values of the matching circuit is undertaken through an optimization framework, where a fixed input RF power is injected via the Agilent N5181 MXG $\mathrm{RF}$ signal generator, and the resulting changes in the output voltage values are measured through the Agilent 34401A multimeter, while sweeping the input frequency of the circuit. After we determine the frequency at which the output voltage value reaches a maxima, we add the capacitor and inductor components on the matching circuit as series and parallel, respectively, to change the frequency of the peak response and draw it closer to $915 \mathrm{MHz}$, which is the RF frequency of the WuTx.

In order to ensure that power transmission from the antenna to the circuit occurs without any waste of energy, we use a fine granularity in the component value selection, i.e., the capacitor value is varied from $0.1 p F$ to $10 p F$ with $0.1 p F$ step size. Similarly, the value of the inductor is changed from $1 n H$ to $10 n H$ with $1 n H$ step size.

After selection of the series components, we repeat a similar procedure to find the proper component values for the parallel connections of the matching network. These iterations finally result in the peak voltage being attained at a frequency very close to $915 \mathrm{MHz}$. The lower left circuit in Fig. 4 is the final fabricated PCB of our energy harvesting module. The PCB is fabricated with FR-4 epoxy glass substrate and has two layers, one of which serves as a ground plane. We select components with values and ratings of their performance parameter as close as possible to the ones obtained from the simulation. This data is summarized in Table I and Table II.

\section{Design of EH-WISP-Mote}

We connected the energy harvesting circuit with the WISPMote to build an EH-WISP-Mote (Enhanced-WISP-Mote), in order to extend the wake-up range of the WISP-Mote. The output of the energy harvester is connected directly to the $V c c$ pin, i.e., the power supply pin, of the WISP, and the ground pin of the energy harvester is connected to the ground pin of the WISP. Thus, the energy harvesting circuit is connected in parallel to the WISP to provide additional energy harvesting capability, hence extending the wake-up range, while retaining the ability to perform ID-based wake-up.

TABLE I

COMPONENTS USED to BUILD THE ENERgy HARVESTER

\begin{tabular}{|l|l|l|l|}
\hline Component & Value & Component & Value \\
\hline Series Capacitor & $0.1 \mathrm{pF}$ & Stage capacitor & $36 \mathrm{pF}$ \\
Parallel Capacitor & $1.0 \mathrm{pF}$ & Diode & HSMS-2852 \\
\hline
\end{tabular}

IV. DESIGN OF REACH-Mote

\section{A. Wake-up Circuit}

The Tmote Sky can be awakened from a low power sleeping mode by an external rising/falling edge. Thus, it is necessary to build a wake-up circuit to trigger the interrupt on the Tmote Sky node. The WISP-Mote and the EH-WISP-Mote 
TABLE II

PARAMETERS USED IN PCB FABRICATION FOR DUAL-STAGE CiRCUit DESIGN

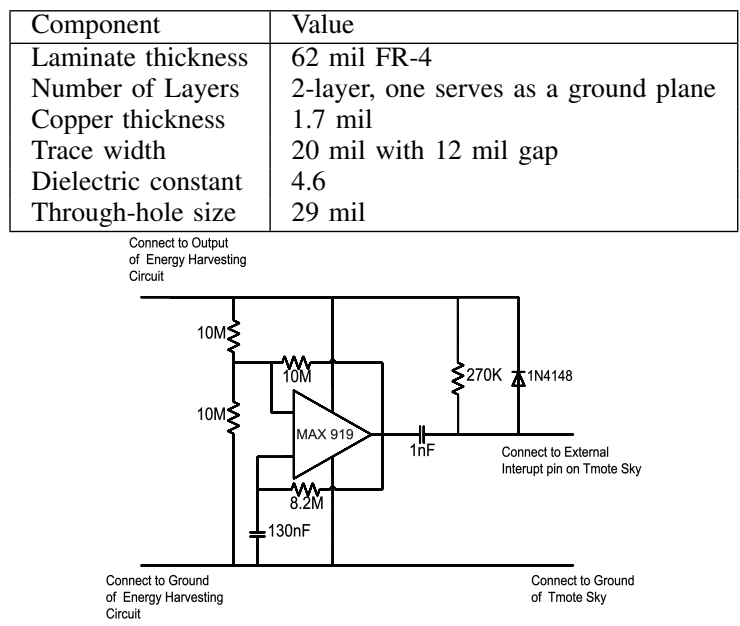

Fig. 3. Wake-up circuit of the REACH-Mote.

use the MCU on the WISP to generate this pulse signal after the WISP receives a wake-up signal. Although the MCU on the WISP has an ultra low power microprocessor, it still consumes $250 \mu \mathrm{A}$ at $2.2 \mathrm{~V}$ power supply. As the amount of energy harvested by the wake-up radio receiver is very limited, the energy consumption of the WISP constrains the wake-up range of the WISP-Mote to approximately $13 f t$ [1]. In order to build a wake-up radio receiver that can operate at an extended range, it is essential for us to build a trigger generator that consumes lower energy than the WISP.

There are several challenges faced in building a trigger circuit, including:

- The circuit must consume very little energy, as the lower the energy consumption, the longer the wake-up range that can be achieved.

- The trigger circuit must generate a rising/falling edge sharp enough to trigger the Tmote Sky wake-up.

- The trigger circuit must work on a variable support voltage as the voltage level output by the energy harvesting circuit is unstable.

Fig. 3 shows the wake-up circuit we built to trigger the Tmote Sky mote [3]. Experimental results show that the circuit can operate at a voltage range from $1.5 \mathrm{~V}$ to $5 \mathrm{~V}$, and it can generate a pulse of $100 \mu s$ duration once per second. Moreover,

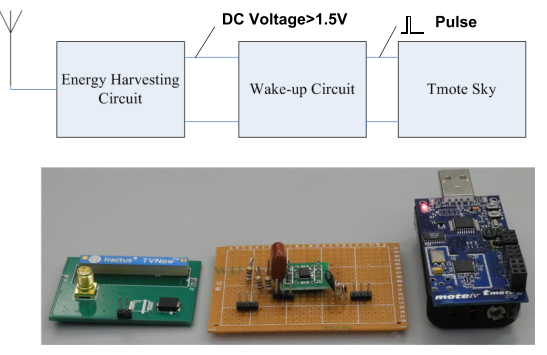

Fig. 4. REACH-Mote system components.

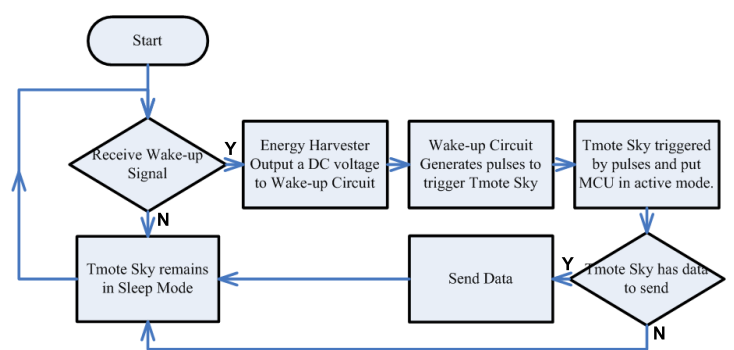

Fig. 5. REACH-Mote flow chart.

the current consumed in this circuit is less than $1 \mu \mathrm{A}$, which is low enough to be driven by the energy harvester output even with very low power reception. Thus this circuit can achieve the design requirements of an ultra low power wake-up radio trigger.

We combine the RF energy harvesting circuit and the wakeup circuit as well as the Tmote Sky to build a new wake-up radio sensor node, which we called a REACH-Mote (Range EnhAnCing energy Harvester-Mote). Fig. 4 shows a block diagram of the REACH-Mote and the actual components. In a wireless sensor network, the REACH-Mote works as described in Fig. 5. The REACH-Mote is put in sleep mode before the WuTx transmits the wake-up signal, i.e., the MCU on the Tmote Sky, which is an MSP430F1611 is put to LPM3 sleep mode [10] and the radio on the Tmote Sky is in the sleep mode. When a wake-up signal is sent by the WuTx, the energy harvesting circuit outputs a DC voltage. The wake-up circuit starts to generate the pulse once the DC voltage is higher than $1.5 \mathrm{~V}$, and this will trigger the mote and put the mote's MCU into active mode in $5 \mathrm{~ms}$ [11]. After waking up, the data transfer is started if the mote has data to send. The REACHMote goes back to the sleep mode after the data transfer is finished.

In this work flow, the energy harvesting circuit is a passive component that does not consume energy from the battery. The wake-up circuit is powered by the energy harvesting circuit, so the wake-up circuit also does not drain energy from the battery. Thus, all of the energy provided by the REACH-Mote battery is used for sensing and data communication.

\section{B. Comparison of the REACH-Mote and the WISP-Mote}

All of the motes we have described (WISP-Mote, EHWISP-Mote and REACH-Mote) can achieve passive radio wake-up. However, there are a few differences among these approaches.

The WISP-Mote and EH-WISP-Mote have the following advantages:

- As the WISP is equipped with a MCU and radio receiving/decoding hardware, the WISP-Mote and EH-WISPMote can achieve ID-based wake-up in cooperation with an RFID reader. This is an advantage over the REACHMote, as ID-based wake-up eliminates wake-ups when the sink is not interested in data from all sensor nodes.

- The WISP's MCU can perform pre-processing for the data received during the wake-up period, which potentially increases the flexibility in MAC protocol design. 
- The WISP's MCU can help to reduce unnecessary wakeups when the sensor node does not have data to send to the WuTx.

Most advantages of the WISP-Mote and the EH-WISP-Mote are a result of the WISP's MCU. However, the MCU also increases the energy consumption of the harvested energy, which may decrease the wake-up radio performance. Consequently, the REACH-Mote has the following advantages as an alternative WuRx solution:

- The monetary cost of the REACH-Mote is lower than the WISP-Mote and the EH-WISP-Mote, as no expensive MCU or decoding components are used.

- As the REACH-Mote wake-up circuit consumes much less energy than the WISP-Mote and the EH-WISPMote, it enables the REACH-Mote to have an advantage in wake-up range and wake-up delay over these other designs.

\section{EXPERIMENTS}

\section{A. Experimental Setup}

In order to compare the performances of the WISP-Mote, the EH-WISP-Mote, and the REACH-Mote, we ran several experiments in an open-space environment (we used an empty gym for these experiments). In our experiments, we mounted the transmitter (WuTx) $2 f t$ above the ground and varied the location of the wake-up receiver (WuRx) in the horizontal (xaxis) and vertical (y-axis) directions with respect to the WuTx. For broadcast-based wake-up, the WuTx transmits a generic wake-up signal that activates any nearby receivers. After the WuRx receives the signal, it wakes up the Tmote Sky, which transmits a short data packet indicating the successful wake-up to the base station (a T-Mote Sky connected to a laptop via USB) located adjacent to the WuTx.

In our experiments, we measure the response delay. For a given WuRx location $(\mathrm{x}, \mathrm{y})$, the WuTx is powered on and an initial time stamp is recorded on a laptop. The WuTx outputs a continuous wake-up signal until the mote wakes up and transmits to the base station, at which point the final time stamp is recorded. The time difference between these time stamps represents the wake-up delay. If the mote does not respond within $100 \mathrm{~s}$, we assume that it cannot be woken up at that particular location.

The wake-up tests are done in $2 f t$ increments in the $\mathrm{x}$ direction starting from $0.1 \mathrm{ft}$ away from the WuTx until the distance after which no wake-up is observed within the delay limit of $100 \mathrm{~s}$. Then the same process is repeated by increasing the vertical distance (y) by $1 \mathrm{ft}$. After each single measurement, the T-Mote Sky is reset using the onboard reset button and the energy harvesting circuit is discharged. For each location, five tests are performed, and the average of these five delay measurements are presented in the following result sections.

\section{B. Results: ID-based Wake-up}

In the first set of experiments, we evaluate the performance of ID-based wake-up for the WISP-Mote and the EH-WISP-

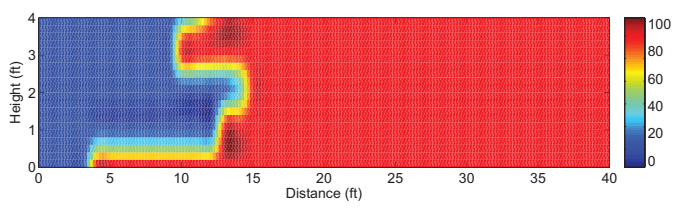

(a) WuTx: RFID Reader and Powercast; WuRx: WISP-Mote

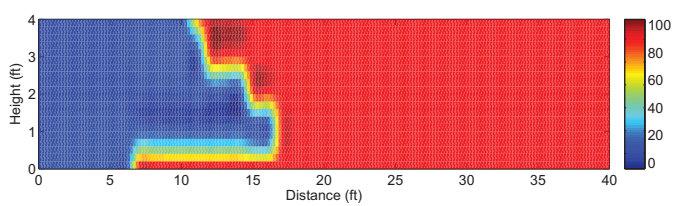

(b) WuTx: RFID Reader and Powercast; WuRx: EH-WISP-Mote

Fig. 6. ID-based wake-up delay (in seconds) for the WISP-Mote and the EHWISP-Mote being woken up by an RFID Reader and Powercast combination. The delay limit of $100 \mathrm{~s}$ is also used to represent the no wake-up locations.

Mote. For the WuTx, we use a combination of an Impinj RFID Reader [7] (which transmits the ID signal that can be read by the WISP) and a Powercast transmitter [12], vertically stacked with their midpoint located $2 f t$ above the ground. The aim of this set of experiments is to evaluate the performance of the EH-WISP-Mote compared with the WISP-Mote, highlighting the advantage of the additional energy harvesting circuit.

Fig. 6 shows the wake-up delay for the EH-WISP-Mote and the WISP-Mote for ID-based wake-up. Comparing the performance of the EH-WISP-Mote and the WISP-Mote, we see that the EH-WISP-Mote can reach $17 f t$ wake-up range at a height of $1 \mathrm{ft}$ above the ground, $3 \mathrm{ft}$ further than the WISP-Mote's maximum wake-up range, representing a $20 \%$ improvement in the maximum wake-up range performance. Additionally, the EH-WISP-Mote doubles the wake-up range when the WuRx is located very close to the ground. Moreover, the total wake-up area of the EH-WISP-Mote is $30 \%$ more than that of the WISP-Mote. These results show that the energy harvesting circuit can provide tangible benefits to the WISPMote, resulting in an improved performance.

\section{Results: Broadcast-based Wake-up}

In this set of experiments, we use broadcast-based wakeup and evaluate the performance of the REACH-Mote, the EH-WISP-Mote and the WISP-Mote. For the WuTx, we use different combinations, including the Impinj RFID Reader [7], the Powercast transmitter [12], and a combination of the two.

Fig. 7 shows the delay results for the different WuTx and WuRx options utilizing broadcast wake-up. From these results, we can see that the wake-up range of the EH-WISP-Mote with any of the WuTxs increases by approximately $20 \%$ compared to the wake-up range of the WISP-Mote. This shows the benefit of adding the energy harvesting circuit for passive wake-up. As the broadcast-based wake-up does not require decoding, the total energy needed is less than that of ID-based wake-ups. Thus, broadcast wake-up achieves longer distances compared to ID-based wake-ups, as can be seen by comparing Fig. 6 with Fig. 7 (c) and Fig. 7(f). Moreover, as seen in Fig. 7(b), the WISP-Mote is observed to not work well with the Powercast WuTx, yielding poor results. The reason is that the Powercast transmitter is not matched to the WISP. 


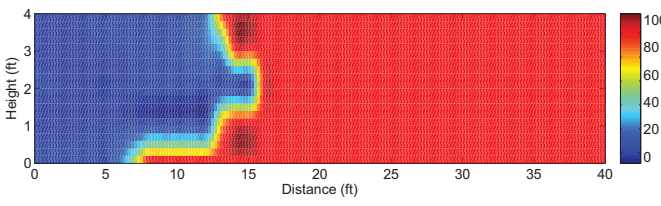

(a) WuTx: RFID Reader; WuRx: WISP-Mote

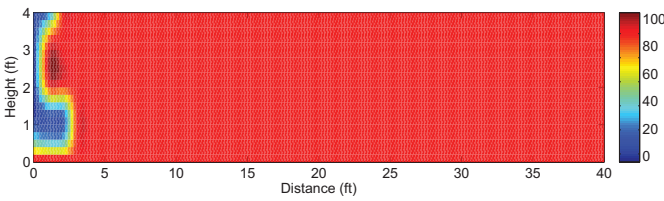

(b) WuTx: Powercast; WuRx: WISP-Mote

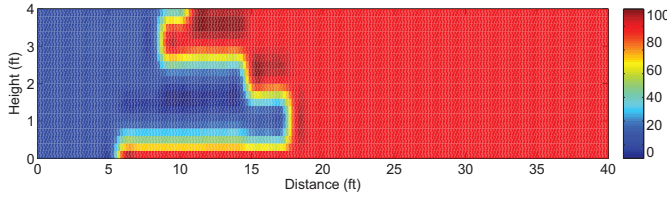

(c) WuTx: RFID Reader and Powercast; WuRx: WISP-Mote

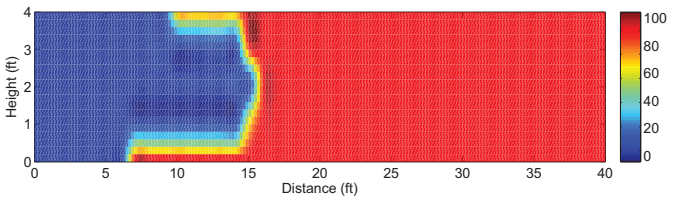

(d) WuTx: RFID Reader; WuRx: EH-WISP-Mote

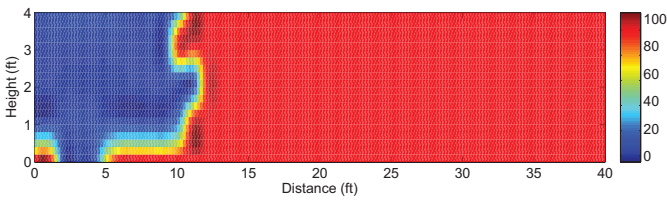

(e) WuTx: Powercast; WuRx: EH-WISP-Mote

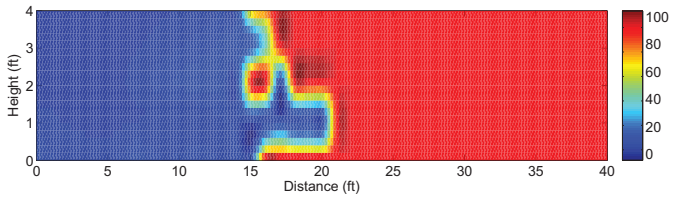

(f) WuTx: RFID Reader and Powercast; WuRx: EH-WISP-Mote

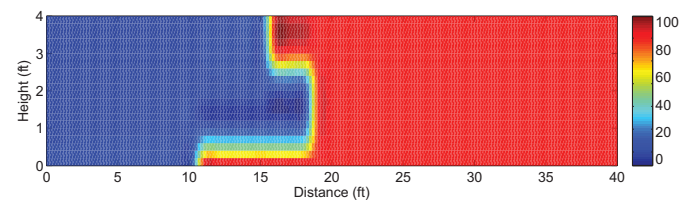

(g) WuTx: RFID Reader; WuRx: REACH-Mote

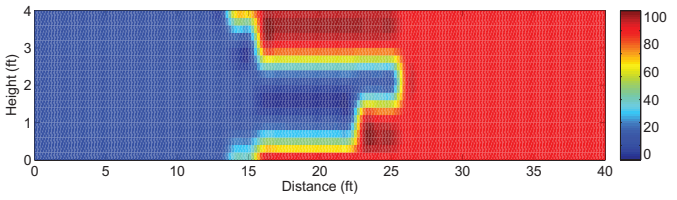

(h) WuTx: Powercast; WuRx: REACH-Mote

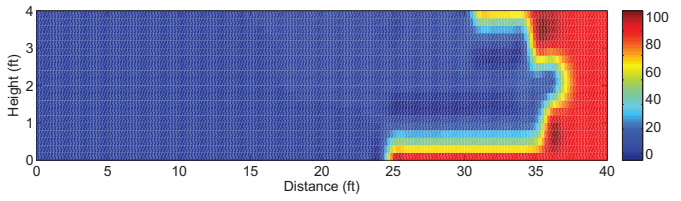

(i) WuTx: RFID Reader and Powercast; WuRx: REACH-Mote

Fig. 7. Wake-up delay (in seconds) for different combinations of WuTxs (RFID Reader, Powercast, and combination of RFID Reader and Powercast) and WuRxs (WISP-Mote, EH-WISP-Mote and REACH-Mote). The delay limit of 100 seconds is also used to represent the no wake-up locations.
The REACH-Mote operating with the combined ReaderPowercast WuTx provides the overall best performance, achieving a wake-up range of $37 \mathrm{ft}$ and covering an area almost $3 \times$ that of the WISP-Mote and $2 \times$ that of the EH-WISPMote. This is due to the ultra low energy consumption of the proposed wake-up circuit. Moreover, the achieved distance represents an impressive range for a battery-less wake-up radio system, i.e., a passive WuRx. To the best of our knowledge, this is the longest wake-up range achieved by a passive wakeup receiver.

In addition, the REACH-Mote achieves the best delay results for any given location. As an example, when these three WuRxs are deployed at $5 \mathrm{ft}$ from the WuTx, the REACHMote can be woken up on average within $235 \mathrm{~ms}$, while the EH-WISP-Mote can be woken up on average within $443 \mathrm{~ms}$. The WISP-Mote has the longest wake-up delay, an average of $721 \mathrm{~ms}$.

\section{CONClusions AND Future Work}

In this paper, we proposed the REACH-Mote, an ultra low power WuRx to improve the wake-up range for passive wake-up radio sensor nodes. Experimental results show that the REACH-Mote doubles the wake-up range compared to the WISP-Mote with different WuTxs. We also implemented an EH-WISP-Mote to increase the wake-up performance of the WISP-Mote. The EH-WISP-Mote retains the same IDbased wake-up capability as the WISP-Mote and can achieve $20 \%$ better coverage than the WISP-Mote. As future work, we plan to evaluate the performance of REACH-Motes in different communication scenarios and compare the benefits and drawbacks of long range broadcast wake-ups achieved with REACH-Motes and shorter range ID-based wake-ups achieved with EH-WISP-Motes.

\section{REFERENCES}

[1] H. Ba, I. Demirkol and W. Heinzelman, "Feasibility and Benefits of Passive RFID Wake-up Radios for Wireless Sensor Networks," IEEE Globecom, 2010.

[2] P. Nintanavongsa, U. Muncuk, D. R. Lewis, K. R. Chowdhury,"Design Optimization and Implementation for RF Energy Harvesting Circuits," JETCAS'12, March 2012

[3] http://www.maximintegrated.com/app-notes/index.mvp/id/1186

[4] M. J. Miller, N. H. Vaidya, "Minimizing energy consumption in sensor networks using a wakeup radio," Wireless Communications and Networking Conference, 2004.

[5] A. Sanchez, J. Aguilar, S. Blanc and J. J. Serrano, "RFID-based wakeup system for wireless sensor networks," VLSI Circuits and Systems V, edited by Teresa Riesgo.

[6] P. Kolinko and L. E. Larson, "Passive RF Receiver Design for Wireless Sensor Networks," Microwave Symposium, 2007.

[7] http://www.impinj.com/

[8] S. Basagni, M. Y. Naderi, C. Petrioli, and D. Spenza."Wireless sensor networks with energy harvesting,'In S. Basagni, M. Conti, S. Giordano, and I. Stojmenovic, editors, Mobile Ad Hoc Networking: Cutting Edge Directions, John Wiley \& Sons, Inc., Hoboken, NJ, 2013.

[9] H. Yan, J. G. Macias Montero, A. Akhnoukh, L. C. N. de Vreede, J. N. Burghart,"An Integration Scheme for RF Power Harvesting,"The 8th Annual Workshop on Semiconductor Advances for Future Electronics and Sensors, Veldhoven, Netherlands, 2005.

[10] http://www.ti.com/product/msp430f1611

[11] http://www.ti.com/tool/msp430-3p-motei-tmotesky-dsgkt

[12] http://www.powercastco.com/ 